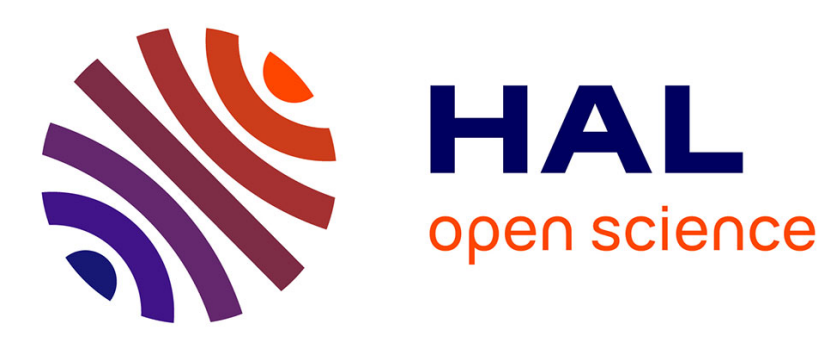

\title{
Discontinuous fluctuation distribution
}

Matthew Hubbard, Mario Ricchiuto

\section{To cite this version:}

Matthew Hubbard, Mario Ricchiuto. Discontinuous fluctuation distribution. [Research Report] RR7402, INRIA. 2010. inria-00522477

\section{HAL Id: inria-00522477 \\ https://hal.inria.fr/inria-00522477}

Submitted on 30 Sep 2010

HAL is a multi-disciplinary open access archive for the deposit and dissemination of scientific research documents, whether they are published or not. The documents may come from teaching and research institutions in France or abroad, or from public or private research centers.
L'archive ouverte pluridisciplinaire HAL, est destinée au dépôt et à la diffusion de documents scientifiques de niveau recherche, publiés ou non, émanant des établissements d'enseignement et de recherche français ou étrangers, des laboratoires publics ou privés. 
INSTITUT NATIONAL DE RECHERCHE EN INFORMATIQUE ET EN AUTOMATIQUE

\title{
Discontinuous fluctuation distribution
}

\author{
Matthew Hubbard — Mario Ricchiuto
}

$\mathbf{N}^{\circ} \mathbf{7 4 0 2}$

April 2010

Thème NUM 



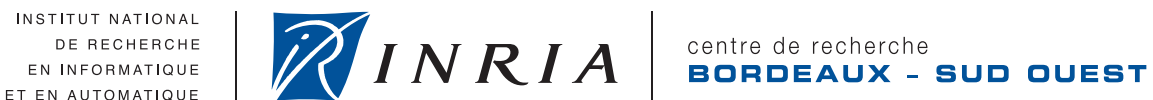

\title{
Discontinuous fluctuation distribution
}

\author{
Matthew Hubbard*, Mario Ricchiutd丹 \\ Thème NUM — Systèmes numériques \\ Équipes-Projets BACCHUS \\ Rapport de recherche $\mathrm{n}^{\circ} 7402$ - April 2010 - 14 pages
}

\begin{abstract}
This paper proposes an approach to the approximation of timedependent hyperbolic conservation laws which is both second order accurate in space and time (for any sufficiently smooth solution profile, even one containing turning points) and free of spurious oscillations for any time-step. The numerical algorithm is based on the concept of fluctuation distribution, applied on a space-time mesh of triangular prisms, for which second order accurate schemes already exist which are oscillation-free if the time-step satisfies a CFL-type constraint. This restriction is lifted here by combining the concept of a two-layer scheme with a representation of the solution which is allowed to be discontinuous in time. Numerical results are presented in two space dimensions, using unstructured meshes of space-time triangular prisms, for the scalar advection equation, Burgers' equation and the Euler equations of gasdynamics.
\end{abstract}

Key-words: conservation laws, positivity, stability, large time steps, residual distribution, second order accuracy

* University of Leeds

$\dagger$ INRIA Bordeaux - Sud-Ouest

Centre de recherche INRIA Bordeaux - Sud Ouest 


\section{Discontinuous residual distribution : A route to unconditional positivity and high order accuracy}

Résumé : This paper proposes an approach to the approximation of timedependent hyperbolic conservation laws which is both second order accurate in space and time (for any sufficiently smooth solution profile, even one containing turning points) and free of spurious oscillations for any time-step. The numerical algorithm is based on the concept of fluctuation distribution, applied on a space-time mesh of triangular prisms, for which second order accurate schemes already exist which are oscillation-free if the time-step satisfies a CFL-type constraint. This restriction is lifted here by combining the concept of a two-layer scheme with a representation of the solution which is allowed to be discontinuous in time. Numerical results are presented in two space dimensions, using unstructured meshes of space-time triangular prisms, for the scalar advection equation, Burgers' equation and the Euler equations of gasdynamics.

Mots-clés : conservation laws, positivity, stability, large time steps, residual distribution, second order accuracy 


\section{Introduction}

The fluctuation distribution framework was developed as an alternative to the finite volume approach for approximating hyperbolic systems of conservation laws which would allow a natural representation of genuinely multidimensional flow features. The resulting algorithms are closely related to conforming finite elements, but their structure makes it far simpler to construct nonlinear approximation schemes, and therefore to avoid unphysical oscillations in the numerical solution. Recent reviews of the state-of-the-art and descriptions of these schemes can be found in [1, 6, 12,

The original formulation, which has been successfully used to simulate steady flow around complete aircraft configurations [8], led naturally to schemes which can achieve second order accuracy in smooth flows without introducing spurious numerical oscillations in the vicinity of discontinuities. Those schemes, however, reduce to first order accuracy away from the steady state, so much recent research has aimed to impose the same combination of properties on time-dependent simulations, leading to the space-time formulation [5] described briefly in Section 2

All of the aforementioned schemes are based on the assumption that the solution varies continuously across the computational domain, but it is straightforward to generalise the framework to allow discontinuities across the faces of the mesh 3, 7]. This additional flexibility provides a number of advantages, e.g. $h$ - and $p$-adaptivity are more easily applied, solution discontinuities can be captured exactly when aligned with the mesh, and characteristic boundary conditions can be implemented in a fully conservative manner. However, for the purposes of this work, the most important property is that allowing the solution to be discontinuous in time means that the distribution scheme used in the space-time cells no longer has to be upwind in time. This removes the CFLlike positivity constraint on the time-step so that a scheme can be constructed which is both second order accurate in space and time and unconditionally positive. This scheme will be summarised in Section 3 and numerical results will be presented in Section 4 .

\section{Space-Time Fluctuation Distribution}

Consider the scalar conservation law governing the evolution of an unknown quantity $u(\vec{x}, t)$ and given by

$$
u_{t}+\vec{\nabla} \cdot \vec{f}=0 \quad \text { or } \quad u_{t}+\vec{\lambda} \cdot \vec{\nabla} u=0
$$

on a domain $\Omega$, with the appropriate initial conditions and Dirichlet boundary conditions imposed on the inflow part of the boundary $\partial \Omega$. Here $\vec{f}$ represents the conservative flux vector and $\vec{\lambda}=\partial \vec{f} / \partial u$ defines the advection velocity associated with the conservation law (1). This equation has an associated fluctuation which, for a space-time mesh cell $C_{j, n}$ (assumed here to be a triangular prism, 
$c f$. the diagram on the left in Figure 10, is given by

$$
\begin{aligned}
\phi_{j, n} & =\int_{C_{j, n}} u_{t}+\vec{\nabla} \cdot \vec{f} d \Omega=\int_{t^{n}}^{t^{n+1}} \int_{C_{j}} u_{t}+\vec{\nabla} \cdot \vec{f} d \vec{x} d t \\
& =\int_{C_{j}} u^{n+1}-u^{n} d \vec{x}+\int_{t^{n}}^{t^{n+1}} \int_{C_{j}} \vec{\nabla} \cdot \vec{f} d \vec{x} d t \\
& =\int_{C_{j}} u^{n+1}-u^{n} d \vec{x}-\int_{t^{n}}^{t^{n+1}} \oint_{\partial C_{j}} \vec{f} \cdot \vec{n} d s d t
\end{aligned}
$$

in which $\vec{n}$ represents the inward pointing unit normal to $\partial C_{j}$, the boundary of the space cell $C_{j}$. Assume now that $u$ is continuous and has a piecewise linear variation in space and in time, and that its discrete values are stored at the nodes of the space-time mesh. The discrete fluctuation $\phi_{j, n}$ is evaluated by combining the midpoint rule in time with exact integration in space (which can be carried out using an appropriate, conservative, linearisation) [5]. This gives a second order accurate representation of the fluctuation which can be written as

$$
\begin{aligned}
\phi_{j, n} & =\sum_{i \in C_{j}} \frac{\left|C_{j}\right|}{3}\left(u_{i}^{n+1}-u_{i}^{n}\right)+\frac{\Delta t}{2} \sum_{i \in C_{j}}\left(k_{i} u_{i}^{n+1}+k_{i} u_{i}^{n}\right) \\
& =\sum_{i \in C_{j}}\left(\frac{\Delta t k_{i}}{2}+\frac{\left|C_{j}\right|}{3}\right) u_{i}^{n+1}+\sum_{i \in C_{j}}\left(\frac{\Delta t k_{i}}{2}-\frac{\left|C_{j}\right|}{3}\right) u_{i}^{n} \\
& =\sum_{i \in C_{j}} \widetilde{k}_{i} u_{i}^{n+1}+\sum_{i \in C_{j}} \widehat{k}_{i} u_{i}^{n} .
\end{aligned}
$$

$\left|C_{j}\right|$ is the area of the triangular space cell $C_{j}, k_{i}=\frac{1}{2} \vec{\lambda} \cdot \vec{n}_{i}$ represent the "inflow" parameters used by the steady state schemes, and $\vec{\lambda}$ is typically evaluated at an appropriate cell-averaged state. The $\widetilde{k}_{i}$ and $\widehat{k}_{i}$ are the space-time "inflow" parameters, i.e. for an upwind scheme in space-time a node is only updated if the corresponding $k_{i}>0$ [5].

The differential equation (1) is approximated at each mesh node by a weighted average of space-time fluctuations from the neighbouring cells. A single timestep then requires the solution of a system of equations, each of which takes the form

$$
\sum_{j, n \mid i \in C_{j, n}} \alpha_{i}^{j, n} \phi_{j, n}=0 \quad \forall \text { nodes } i .
$$

This could be solved directly, but in this work a pseudo-time-stepping algorithm is applied at each time level $n$,

$$
\left(u_{i}^{n+1}\right)^{(m+1)}=\left(u_{i}^{n+1}\right)^{(m)}+\frac{\Delta \tau}{\left|\Omega_{i, n}\right|} \sum_{j, n \mid i \in C_{j, n}} \alpha_{i}^{j, n} \phi_{j, n}
$$

in which $\left|\Omega_{i, n}\right|=\frac{1}{6} \Delta t \sum_{j \mid i \in C_{j}}\left|C_{j}\right|$, the volume of the space-time median dual cell associated with node $i$ restricted to the layer starting at time level $n$, acts purely as a relaxation parameter.

The properties of a scheme depend on the precise choice of the distribution coefficients $\alpha_{i}^{j, n}$ or, equivalently, $\phi_{i}^{j, n}=\alpha_{i}^{j, n} \phi_{j, n}$, the contributions from cell $j, n$ to node $i$ [12]. 

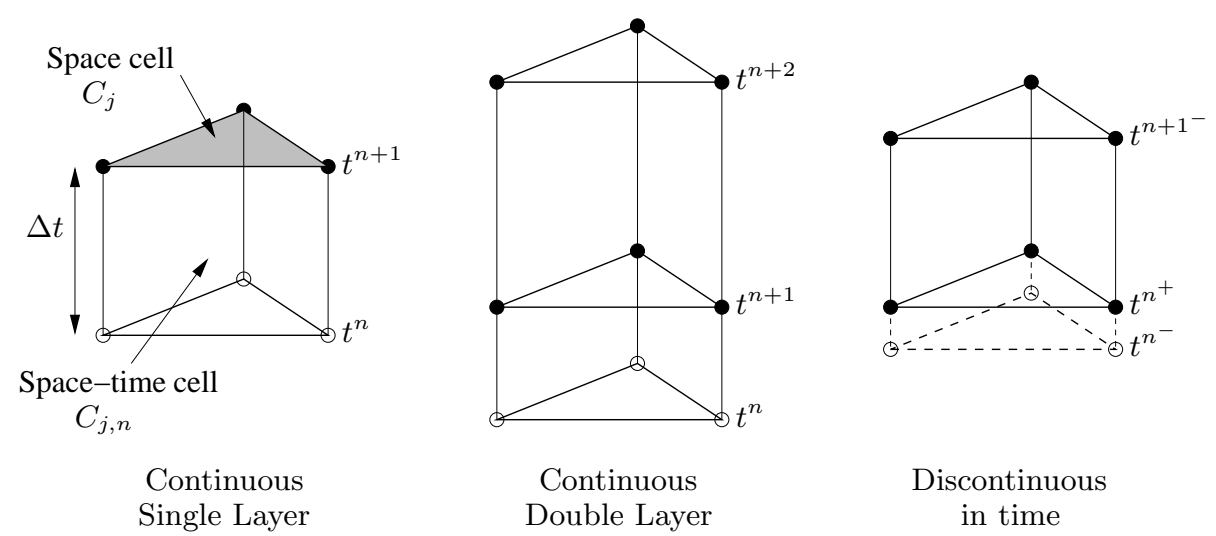

Figure 1: Space-time cell configurations for the different forms of scheme: indicates the unknown solution values when solving for a given time-step; o indicates the known solution values.

Positivity ensures that the numerical approximations are free of unphysical oscillations by forcing each solution value at time level $n+1$ to be a positive combination of solution values at time level $n$. This has traditionally imposed a CFL-like condition on the time-step.

Linearity preservation ensures that the distribution of a fluctuation evaluated exactly with respect to a $(k-1)^{\text {th }}$ degree polynomial representation of the flux will lead to a $k^{\text {th }}$ order accurate scheme 2]. It is assured as long as the distribution coefficients $\alpha_{i}^{j, n}$ are bounded.

Conservation ensures that discontinuities are captured correctly, and is assured as long as, for every cell $C_{j, n}, \sum_{i \in C_{j, n}} \alpha_{i}^{j, n}=1$, i.e. the whole of each fluctuation is distributed.

Compactness aids the efficiency of the algorithm, especially when parallelisation is considered. For piecewise linear variation of $u$ this simply restricts the schemes so that a cell's fluctuation is only distributed to its own vertices.

Continuous dependence of the distribution coefficients on both the dependent variable and the advection velocity facilitates smooth iterative convergence at each time level.

Upwinding dictates that the discrete form should propagate signals in the same directions and with the same speeds as those inherent in the mathematical/physical model. This facilitates the construction of positive schemes, provides rapid and smooth iterative convergence, and simplifies the imposition of boundary conditions.

In line with Godunov's theorem, linear fluctuation distribution schemes cannot be both positive and linearity preserving. However, they can be used as the basis for developing nonlinear schemes which satisfy all of the above properties. 
The N scheme 10] is a linear scheme with all of the desired properties except linearity preservation. It has many equivalent forms, the most useful for the purposes of this work being given by

$$
\left(\phi_{i}^{j, n}\right)^{N}=\Delta t \widehat{k}_{i}^{+}\left(u_{i}^{n}-\widetilde{u}_{i n}\right) \quad\left(\phi_{i}^{j, n+1}\right)^{N}=\Delta t \widetilde{k}_{i}^{+}\left(u_{i}^{n+1}-\widetilde{u}_{i n}\right)
$$

where

$$
\widetilde{u}_{i n}=-\widetilde{N} \sum_{i \in C_{j}}\left(\widetilde{k}_{i}^{+} u_{i}^{n+1}+\widehat{k}_{i}^{+} u_{i}^{n}-\phi_{j, n}\right) \quad \widetilde{N}=\left(\sum_{i \in C_{j}}\left(\widetilde{k}_{i}^{+}+\widehat{k}_{i}^{+}\right)\right)^{-1}
$$

A linear, linearity-preserving (but not positive) scheme is the LDA scheme 10, defined by

$$
\left(\phi_{i}^{j, n}\right)^{L D A}=\widehat{k}_{i}^{+} \tilde{N} \phi_{j, n} \quad\left(\phi_{i}^{j, n+1}\right)^{L D A}=\widetilde{k}_{i}^{+} \tilde{N} \phi_{j, n} .
$$

In order to obtain all of the properties a nonlinear scheme must be constructed. This is often done by "limiting" the coefficients of the $\mathrm{N}$ scheme to impose linearity preservation, e.g.

$$
\alpha_{i}^{j, n}=\frac{\max \left(0,\left(\alpha_{i}^{j, n}\right)^{N}\right)}{\sum_{k \in C_{j, n}} \max \left(0,\left(\alpha_{k}^{j, n}\right)^{N}\right)} \quad\left(\alpha_{i}^{j, n}\right)^{N}=\frac{\left(\phi_{i}^{j, n}\right)^{N}}{\phi_{j, n}}
$$

produces the PSI scheme [11, but a more flexible alternative is to blend two schemes, such as $\mathrm{N}$ and LDA, i.e.

$$
\phi^{B}=\theta \phi^{N}+(1-\theta) \phi^{L D A} \quad \theta \in[0,1] .
$$

In this work, $\theta=\left|\phi_{j, n}\right| / \sum_{i \in C_{j}}\left|\left(\phi_{i}^{j, n}\right)^{N}\right|$ is chosen [8].

Remark: In order to satisfy the "past-shield" condition, the time-step must be chosen so that $\widehat{k}_{i} \leq 0$, which ensures that the distribution is always upwind in time. Otherwise some of the fluctuation would be distributed back to time level $n$ and therefore ignored in the solution update, so the scheme would not be consistent.

\section{$2.1 \quad$ Two-Layer Schemes}

When a continuous representation of the solution is assumed the past-shield condition can be relaxed by the use of a pair of time layers [5]. If a second layer is introduced (see the diagram in the middle of Figure 10) and solved for at the same time as the first layer then it is only necessary to enforce upwinding in time in the first layer. This means that the space-time $\mathrm{N}$ scheme can be used consistently, while remaining positive, for any value of $\Delta t$ in the second layer. It can therefore be combined with the space-time LDA scheme to produce a scheme which is linearity preserving and positive for any time-step, at the expense of doubling the number of unknowns in the system of equations solved at each time-step. 


\section{Discontinuous Fluctuation Distribution}

The fluctuation distribution concept can also be applied when the representation of the solution is allowed to be discontinuous. This simply introduces additional fluctuations derived from the jumps across the space-time mesh cell faces 7 . If each face $F_{k, n}$ is considered to be the limit of a prismatic cell as its breadth tends to zero then the corresponding fluctuations can be written

$$
\psi_{k, n}=\lim _{\epsilon \rightarrow 0} \int_{\Omega_{k, n}} u_{t}+\vec{\nabla} \cdot \vec{f} d \Omega=\int_{F_{k, n}}\left[\vec{f}^{t} \cdot \vec{n}^{t}\right] d \Gamma
$$

in which $\vec{f}^{t}=(u, \vec{f})$ and $\vec{n}^{t}=(1, \vec{n})$ are vectors in space-time. This leads to a separate equation for each cell vertex $i$ of each cell $j, n$, which involves both cell-based and face-based fluctuations and takes the form

$$
\alpha_{i}^{j, n} \phi_{j, n}+\sum_{k, n \mid i \in F_{k, n}} \beta_{i}^{k, n} \psi_{k, n}=0 \quad \forall \text { cell vertices } i \text {. }
$$

In this paper, only discontinuities in time will be considered (see the diagram on the right of Figure 1). The fluctuation across the lower face of the space-time cell $C_{k, n}$, which corresponds to the space cell $C_{k}$ at time $t^{n}$, can be written as

$$
\psi_{k, n}=\int_{C_{k}}\left[\vec{f}^{t} \cdot \vec{n}^{t}\right] d \Gamma=\left|C_{k}\right|[\widetilde{u}]=\left|C_{k}\right|\left(\widetilde{u}^{n^{+}}-\widetilde{u}^{n^{-}}\right)
$$

where $\widetilde{u}$ here is the arithmetic mean of the values of $u$ at the vertices cell $C_{k}$. A positive distribution of this fluctuation which preserves linearity preservation in the overall scheme [7, is given by

$$
\psi_{i}^{k, n}=\beta_{i}^{k, n} \psi_{k, n}=\frac{1}{3}\left|C_{k}\right|\left(u_{i}^{n^{+}}-u_{i}^{n^{-}}\right)
$$

Remark: This is equivalent to a two-layer scheme in which the first layer has degenerated in to a prism of height zero. However, the form of the fluctuation (13) in this first layer and its distribution (14) is much simpler because it no longer contains any contribution from the spatial flux terms.

\section{Numerical Results}

\subsection{Scalar Linear Equations}

The first test case presented here is used to demonstrate the order of accuracy of the algorithm. The scalar advection equation with constant advection in the $x$-direction, i.e.

$$
u_{t}+u_{x}=0
$$

is approximated on the spatial domain $[0,1] \times[0,1]$ with initial state

$$
u_{0}(x, y)= \begin{cases}\cos ^{2}(2 \pi r) & \text { if } r \leq 0.25 \\ 0 & \text { otherwise }\end{cases}
$$

with $r^{2}=(x-0.5)^{2}+(y-0.5)^{2}$. Periodic boundary conditions are imposed on the left and right boundaries. The problem is solved until the final time $T=1$ on 
a set of 4 unstructured triangulations with the topology shown on figure 2, The finer ones are obtained from the coarsest via 3 steps of conformal refinement. Figure 3 clearly shows that close to second order accuracy is achieved even with CFL numbers as high as 50. The blended LDA-N solutions are also free of spurious oscillations.

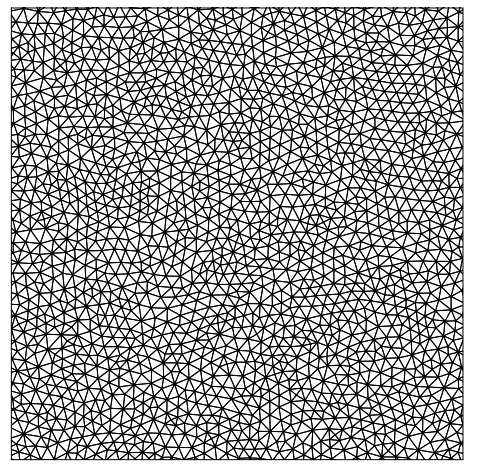

Figure 2: Triangulation used for the scalar test cases.
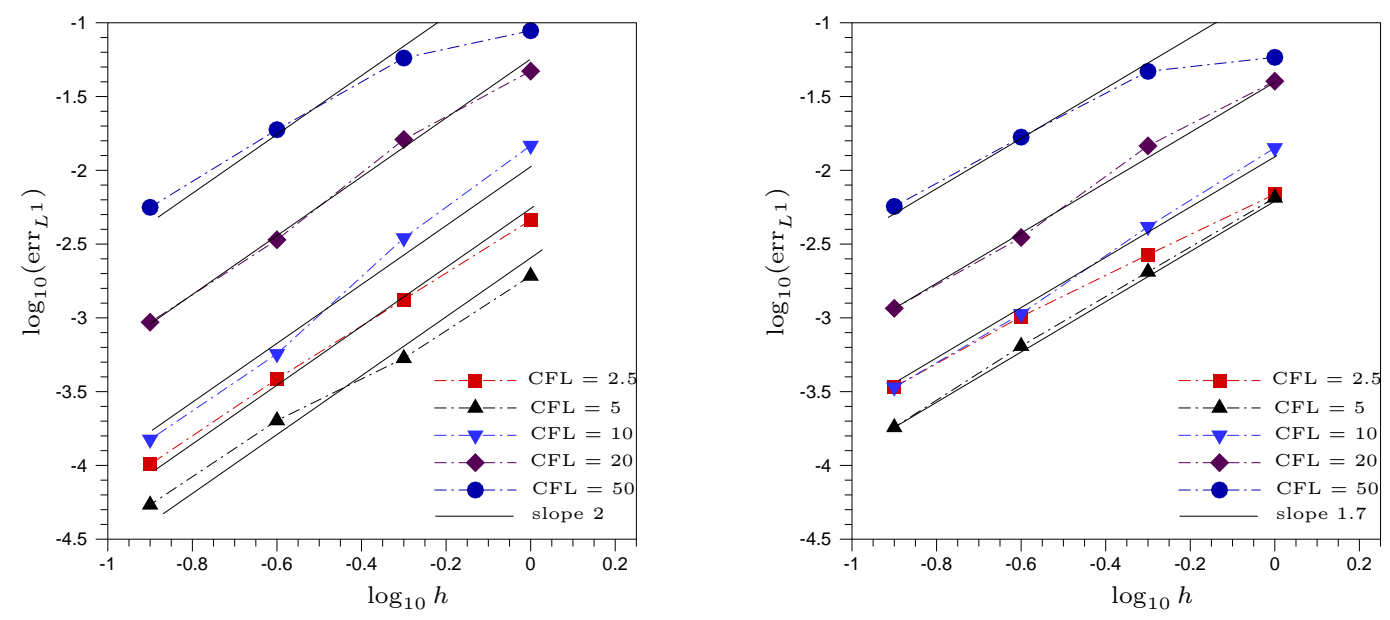

Figure 3: Grid convergence for advection of a smooth profile using continuousin-space, discontinuous-in-time schemes: $L^{1}$ error for the LDA scheme (left); $L^{1}$ error for the LDA-N scheme (right).

\subsection{Scalar Nonlinear Equations}

The second scalar test case presented approximates the two-dimensional Burgers' equation

$$
u_{t}+\left(\frac{u^{2}}{2}\right)_{x}+\left(\frac{u^{2}}{2}\right)_{y}=0
$$


on the spatial domain $[-1,1] \times[-1,1]$ with initial solution

$$
u_{0}(x, y)= \begin{cases}1 & \text { if }(x, y) \in[-0.6,-0.1] \times[-0.35,0.15] \\ 0 & \text { otherwise }\end{cases}
$$

The final time of the simulation is $T=1$ and the problem is solved with the blended LDA-N scheme on an unstructured triangulation of the domain similar to the one shown on figure 2, and with $h=1 / 40$. Figure 4 shows that the solution remains free of oscillations close to the discontinuity.
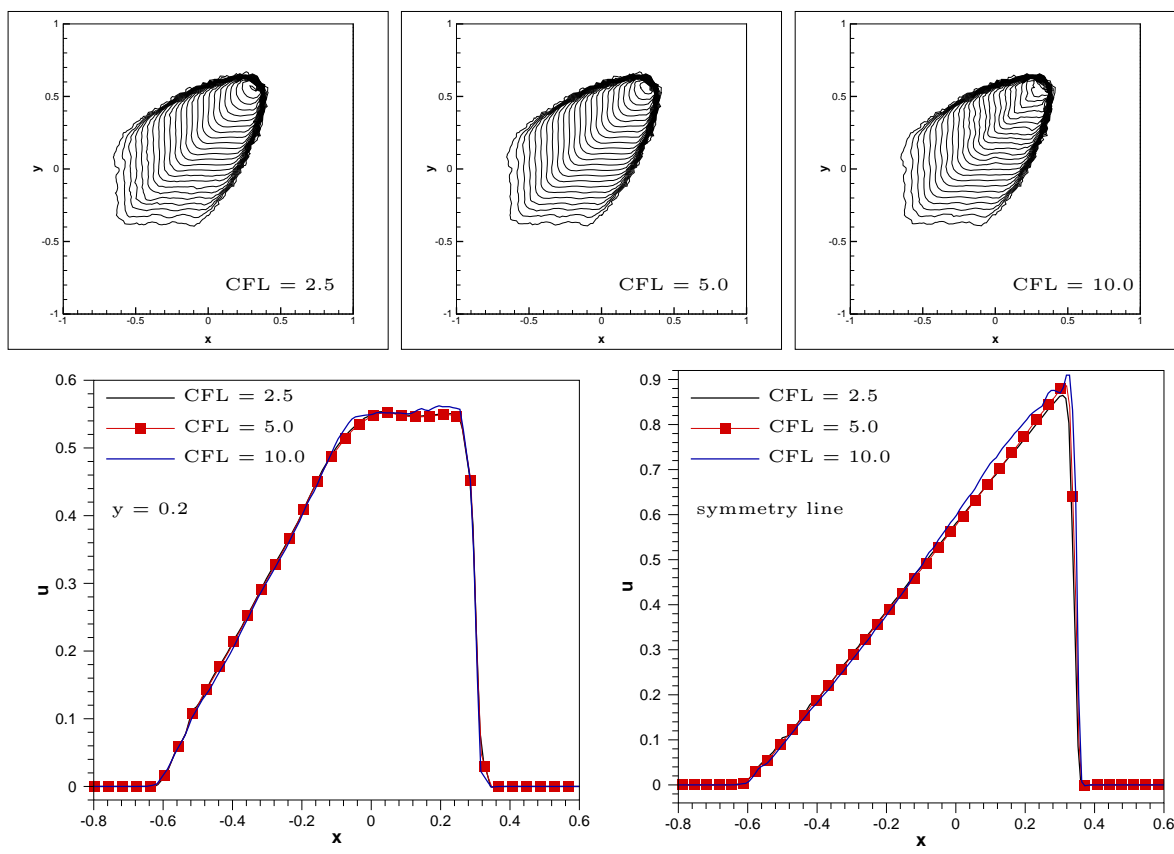

Figure 4: Solutions to the two-dimensional Burgers' equation using the continuous-in-space, discontinuous-in-time LDA-N scheme: contour plots of the solutions obtained with $\mathrm{CFL}=2.5$ (top left), $\mathrm{CFL}=5.0$ (top middle), and $\mathrm{CFL}=10.0$ (top right); data extracted along the line $y=0.2$ (bottom left), and along the symmetry line (bottom right).

\subsection{Nonlinear Systems of Equations}

The approaches described in Sections 2 and 3 can also be applied to nonlinear systems of equations. The fluctuations $\underline{\Phi}$ are still formed at each time level by integrating the conservation laws over each prismatic space-time mesh cell in that time slice [6], i.e.

$$
\underline{U}_{t}+\vec{\nabla} \cdot \underline{\vec{F}}=0 \quad \longrightarrow \quad \underline{\Phi}_{j, n}=\int_{C_{j, n}} \underline{U}_{t}+\vec{\nabla} \cdot \underline{\vec{F}} d \vec{x} d t
$$

but the distribution of this fluctuation is slightly more complicated because the upwind directions associated with the system are not immediately apparent. 
The most commonly-used solution has been to apply a matrix distribution scheme [13. In the space-time cells, analogous to Equation (3),

$$
\underline{\Phi}_{j, n}=\sum_{i \in C_{j, n}} \mathbf{K}_{i} \underline{U}_{i} \quad \text { where } \quad \mathbf{K}_{i}=\frac{\Delta t}{4} \widetilde{\overrightarrow{\mathbf{A}}} \cdot \vec{n}_{i} \pm \frac{\left|C_{j}\right|}{3} \mathbf{I},
$$

in which $\widetilde{\overrightarrow{\mathbf{A}}} \approx \partial \underline{\vec{F}} / \partial \underline{U}$ represents the flux Jacobians, evaluated at an appropriate cell-averaged state, often derived from a conservative linearisation. The fluctuations due to the discontinuities in time are simply

$$
\underline{\Psi}_{k, n}=\frac{\left|C_{k}\right|}{3} \sum_{i \in C_{k}}\left(\underline{U}_{i}^{n^{+}}-\underline{U}_{i}^{n^{-}}\right),
$$

cf. Equations (13) and (14).

The N scheme and LDA scheme can be defined precisely as before, Equations (6) and (8) respectively, except the scalar inflow parameters $k_{i}$ are replaced by the inflow matrices $\mathbf{K}_{i}=\frac{1}{2} \widetilde{\overrightarrow{\mathbf{A}}} \cdot \vec{n}$. These matrices can always be diagonalised for the Euler equations, which leads to $\mathbf{K}^{ \pm}=\mathbf{R} \boldsymbol{\Lambda}^{ \pm} \mathbf{R}^{-1}$, where $\boldsymbol{\Lambda}$ is the diagonal matrix of eigenvalues of $\mathbf{K}$, allowing the application of upwinding componentby-component. The two schemes can then be blended, $c f$. Equation (10), in order to obtain accurate, oscillation-free, numerical approximations.

As in the scalar case, fhe fluctuation due to the space-time discontinuity is distributed in a very simple, pointwise manner, i.e. the contribution from face $j$ to node $i, n^{+}$is given by

$$
\underline{\Psi}_{k}^{i, n^{-}}=0 \quad \underline{\Psi}_{k}^{i, n^{+}}=\frac{\left|C_{k}\right|}{3}\left(\underline{U}_{i}^{n^{+}}-\underline{U}_{i}^{n^{-}}\right) .
$$

Preliminary results obtained using the blended LDA-N scheme on the Mach 3 wind tunnel with a step test case 4 are shown in Figure 6. A zoom of the mesh used for simulation is also shown in Figure 5. The grid is refined close to the corner sigularity, the characteristic size of the elements going from $1 / 80$ to $1 / 1000$ at the singularity. To compensate for the refinement, we have set the CFL to $1000 / 80=12.5$. The contours and the line plots demonstrate that the solution remains free of oscillations for CFL numbers much higher than one. Note that in the system case, we perform the blending on the scalar residual obtained by projecting the vector quantities along characteristic directions, as described in [2].

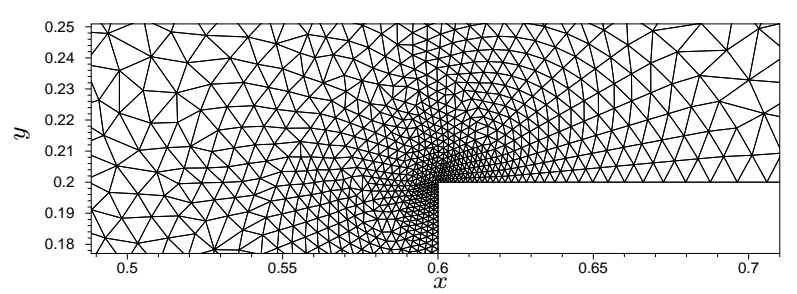

Figure 5: Supersonic backward facing step test case. Close up view of the mesh. 

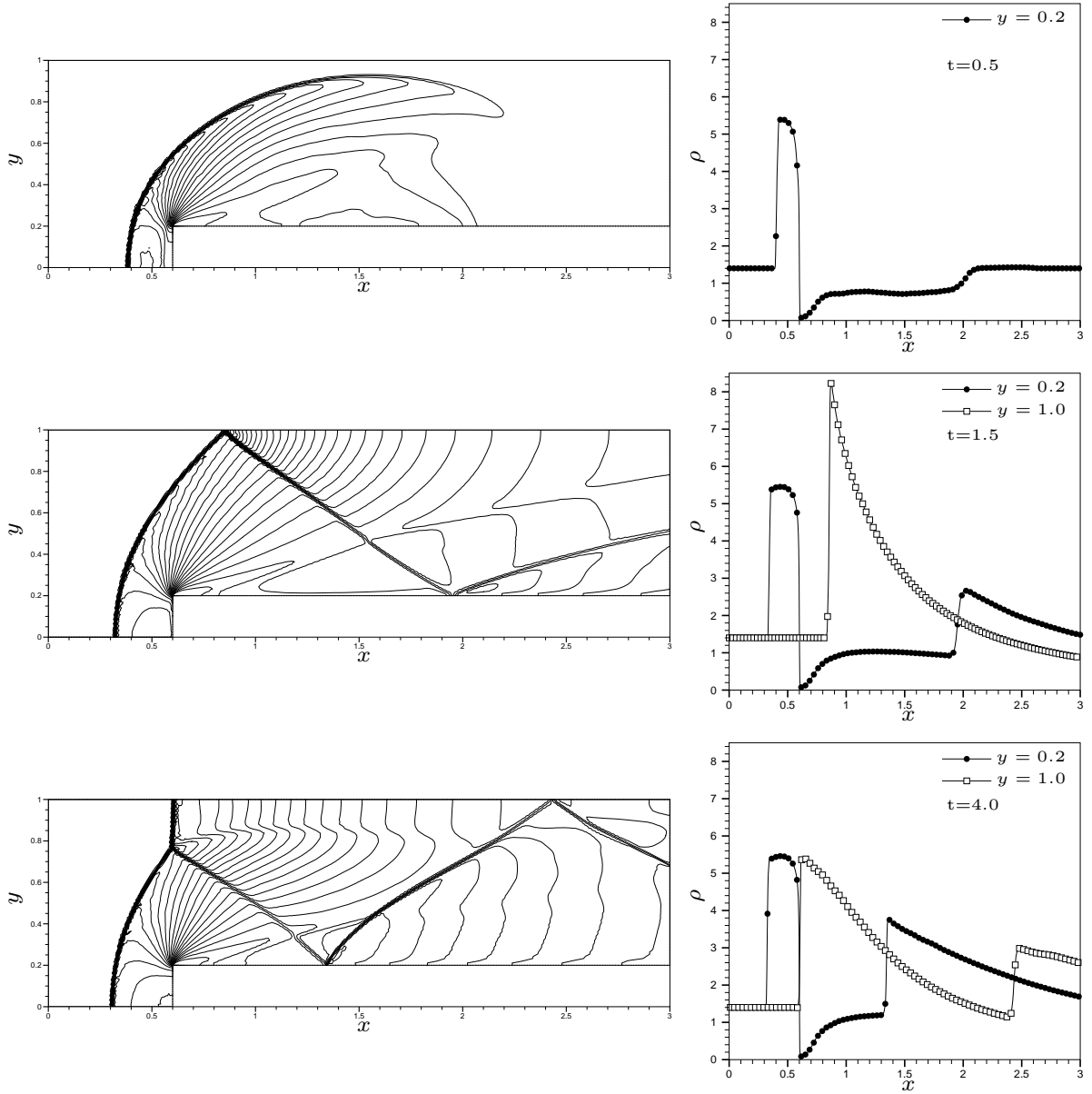

Figure 6: Supersonic backward facing step test case. Density contours, and density distribution along the lines $y=0.2$ (containing the conrner singularity), and $y=1.0$ (upper wall) at times 0.5 (top), 1.5 (middle), and 4.0 (bottom). Flow is from left to right with $M_{\infty}=3.0$ and the CFL is 12.5 . 
The accuracy of the LDA and LDA-N scheme has been also tested on the constant density vortex advection problem proposed in [9. Grid convergence plots for different CFL values are reported in Figure 7 . In the figures we plot the $L^{2}$ norm of the pressure error. As in the scalar case, the computations have been run on triangularions similar to the one shown in Figure 2, the finer grids having been obtained by successive steps of conformal refinement. For the LDA-N scheme one more grid has been used to better verify the behavior of the scheme. The results show that also for the Euler equations, second or almost second order of accuracy is achieved for all CFL numbers. Clearly, the nonlinear scheme has a much larger error and the slopes can be improved. This shows that better definitions of the blanding parameter have to be investigated.
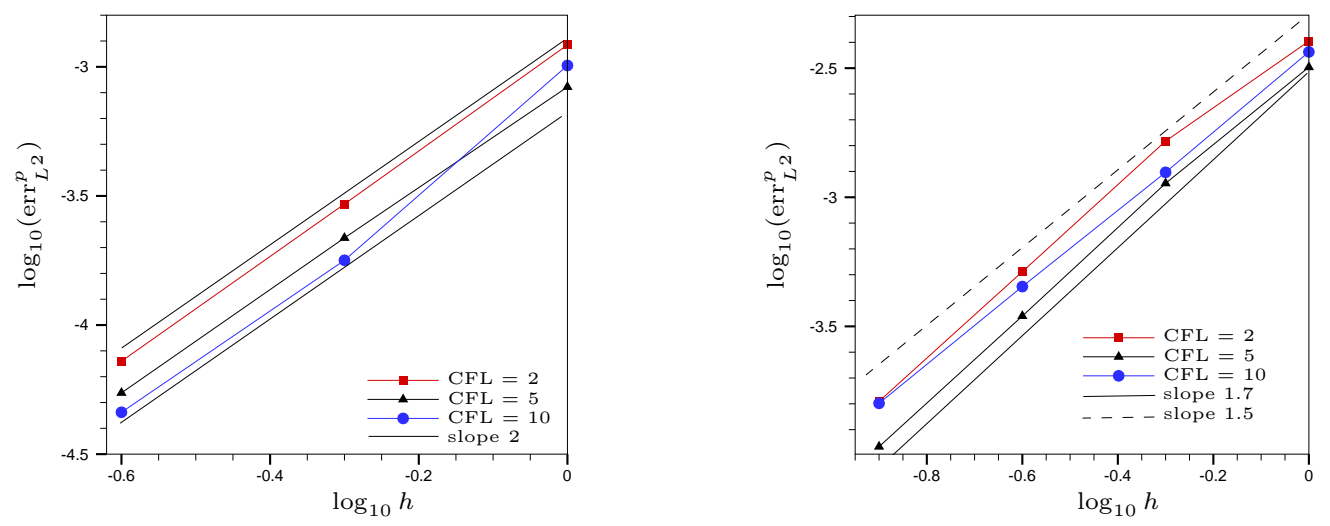

Figure 7: Grid convergence for the constant density vortex advection problem : $L^{2}$ pressure error for the LDA scheme (left); $L^{2}$ pressure error for the LDA-N scheme (right).

\section{Conclusions}

A new formulation has been presented for fluctuation distribution schemes which based on a solution representation which is discontinuous in time. This formulation allows arbitrarily large time-step to be taken without introducing unphysical oscillations in to the numerical solution. It has also been shown that for scalar linear problems with smooth initial conditions the scheme is second order accurate.

The penalty incurred with respect to a standard implicit discretization is the doubling of the number of unknowns/nonlinear equations to be solved at each time step. Work remains in assessing the efficiency of the approach with respect to the accuracy versus computational time.

\section{References}

[1] R.Abgrall, Residual distribution schemes: Current status and future trends, Comput. Fluids, 35:641-669, 2006. 
[2] R.Abgrall, M.Mezine, Construction of second order accurate monotone and stable residual distribution schemes for unsteady flow problems, J. Comput. Phys., 188:16-55, 2003.

[3] R.Abgrall, C.-W.Shu, Development of residual distribution schemes for the Discontinuous Galerkin methods: The scalar case with linear elements, Commun. Comput. Phys., 5:376-390, 2009.

[4] P.R. Woodward, P. Colella, The numerical simulation of two-dimensional flows with strong shocks, J. Comput. Phys., 54:115-1735, 1984.

[5] A.Csik, H.Deconinck, Space-time residual distribution schemes for hyperbolic conservation laws on unstructured linear finite elements, Int. J. Numer. Meth. Fluids, 40:573-581, 2002.

[6] H.Deconinck, M.Ricchiuto, Residual distribution schemes: Foundation and analysis, Encyclopedia of Computational Mechanics, Volume 3: Fluids, Wiley, 2007.

[7] M.E.Hubbard, Discontinuous fluctuation distribution, J. Comput. Phys., 227(24): 10125-10147, 2008.

[8] H.Deconinck, K.Sermeus, R.Abgrall, Status of multidimensional upwind residual distribution schemes and applications in aeronautics, AIAA Paper 2000-2328, AIAA, 2000.

[9] J. Dobes, H. Deconinck, Second order blended multidimensional upwind residual distribution scheme for steady and unsteady computations, $J$. Comput. Appl. Math.. 215: 378-389, 2006.

[10] P.L.Roe, Linear advection schemes on triangular meshes, Technical Report CoS 8720, Cranfield Institute of Technology, 1987.

[11] R.Struijs, H.Deconinck, P.de Palma, P.L.Roe, K.G.Powell, Progress on multidimensional upwind Euler solvers for unstructured grids, AIAA paper, 91-1550, 1991.

[12] High Order Discretization Methods, 34th Computational Fluid Dynamics course, von Karman Institute for Fluid Dynamics, Lecture Series, November 2005 .

[13] E.van der Weide, H.Deconinck, Positive matrix distribution schemes for hyperbolic systems, in Computational Fluid Dynamics, pages 747-753, Wiley, New York, 1996.

\section{Contents}

1 Introduction 3

2 Space-Time Fluctuation Distribution 3

2.1 Two-Laver Schemes . . . . . . . . . . . . . . . . 6

\begin{tabular}{|lll}
\hline & Discontinuous Fluctuation Distribution & 7
\end{tabular} 
\begin{tabular}{|lll}
4 & Numerical Results & 7
\end{tabular}

$4.1 \quad$ Scalar Linear Equations . . . . . . . . . . . . . . . . . . . . . . 7

4.2 Scalar Nonlinear Equations . . . . . . . . . . . . . . . . . . 8

4.3 Nonlinear Systems of Equations . . . . . . . . . . . . . . . . 9

$\begin{array}{lll}5 & \text { Conclusions } & 12\end{array}$ 
Centre de recherche INRIA Bordeaux - Sud Ouest

Domaine Universitaire - 351, cours de la Libération - 33405 Talence Cedex (France)

Centre de recherche INRIA Grenoble - Rhône-Alpes : 655, avenue de l'Europe - 38334 Montbonnot Saint-Ismier

Centre de recherche INRIA Lille - Nord Europe : Parc Scientifique de la Haute Borne - 40, avenue Halley - 59650 Villeneuve d'Ascq

Centre de recherche INRIA Nancy - Grand Est : LORIA, Technopôle de Nancy-Brabois - Campus scientifique 615, rue du Jardin Botanique - BP 101 - 54602 Villers-lès-Nancy Cedex

Centre de recherche INRIA Paris - Rocquencourt : Domaine de Voluceau - Rocquencourt - BP 105 - 78153 Le Chesnay Cedex

Centre de recherche INRIA Rennes - Bretagne Atlantique : IRISA, Campus universitaire de Beaulieu - 35042 Rennes Cedex

Centre de recherche INRIA Saclay - Île-de-France : Parc Orsay Université - ZAC des Vignes : 4, rue Jacques Monod - 91893 Orsay Cedex

Centre de recherche INRIA Sophia Antipolis - Méditerranée : 2004, route des Lucioles - BP 93 - 06902 Sophia Antipolis Cedex 